\title{
Evaluation of a mobile application to support HIV self-testing in Johannesburg, South Africa
}

\begin{tabular}{|c|c|}
\hline $\begin{array}{l}\text { Authors: } \\
\text { Natasha Gous } \\
\text { Alex E. Fischer } \\
\text { Naleni Rhagna } \\
\text { Mothepane Pr } \\
\text { Mohammed N } \\
\text { Samanta T. Lal }\end{array}$ & $\begin{array}{l}\text { (1) } \\
2 \text { (1) } \\
\text { th }^{2} \text { (1) } \\
\text { atsoane } \\
\text { lajam }^{2} \text { (1) } \\
\text { la-Edward }^{2} \text { (1) }\end{array}$ \\
\hline $\begin{array}{l}\text { Affiliations: } \\
{ }^{1} \text { SystemOne, L } \\
\text { Weltevreden } \\
\text { South Africa }\end{array}$ & $\begin{array}{l}\text { LC, } \\
\text { ark, }\end{array}$ \\
\hline $\begin{array}{l}{ }^{2} \text { Ezintsha, Wits } \\
\text { Health and HIV } \\
\text { University of th } \\
\text { Witwatersran } \\
\text { Johannesurg, }\end{array}$ & $\begin{array}{l}\text { Reproductive } \\
\text { Institute, } \\
\text { he } \\
\text { South Africa }\end{array}$ \\
\hline $\begin{array}{l}\text { Corresponding } \\
\text { Alex Fischer, } \\
\text { afischer@wrhi }\end{array}$ & $\begin{array}{l}\text { g author: } \\
\text { i.ac.za }\end{array}$ \\
\hline $\begin{array}{l}\text { Dates: } \\
\text { Received: } 25 \mathrm{~N} \\
\text { Accepted: } 23 \\
\text { Published: } 30\end{array}$ & $\begin{array}{l}\text { Mar. } 2020 \\
\text { Apr. } 2020 \\
\text { June } 2020\end{array}$ \\
\hline $\begin{array}{l}\text { How to cite th } \\
\text { Gous N, Fische } \\
\text { Rhagnath N, P } \\
\text { Majam M, Lall } \\
\text { Evaluation of a } \\
\text { application to } \\
\text { self-testing in } J \\
\text { South Africa. S } \\
2020 ; 21(1): a 10 \\
\text { doi.org/10.41C } \\
\text { V21i1.1088 }\end{array}$ & $\begin{array}{l}\text { is article: } \\
\text { r AE, } \\
\text { hatsoane M, } \\
\text { a-Edward ST. } \\
\text { mobile } \\
\text { support HIV } \\
\text { Johannesburg, } \\
\text { Afr J HIV Med. } \\
\text { 088. https:// } \\
\text { O2/sajhivmed. }\end{array}$ \\
\hline $\begin{array}{l}\text { Copyright: } \\
\text { ๔ 2020. The A } \\
\text { Licensee: AOSI } \\
\text { is licensed und } \\
\text { Creative Comm } \\
\text { Attribution Lice }\end{array}$ & $\begin{array}{l}\text { uthors. } \\
\text { IS. This work } \\
\text { ler the } \\
\text { nons } \\
\text { ense. }\end{array}$ \\
\hline 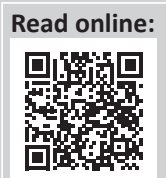 & $\begin{array}{l}\text { Scan this QR } \\
\text { code with your } \\
\text { smart phone or } \\
\text { mobile device } \\
\text { to read online. }\end{array}$ \\
\hline
\end{tabular}

Background: Human immunodeficiency virus self-testing (HIVST) reduces barriers associated with facility-based testing; however, no formal mechanism exists for users to self-report results or link to care. The Aspect ${ }^{\mathrm{TM}}$ HIVST mobile application (app) was developed for use in South Africa.

Objectives: This study evaluated the acceptability and feasibility of the Aspect ${ }^{\mathrm{TM}}$ HIVST app for individuals from the inner city of Johannesburg.

Method: This cross-sectional pilot, with a convenience sample of 300 adults, was conducted in July 2018. Participants were provided an OraQuick HIVST kit and a smartphone preloaded with the app, then asked to follow the in-app instructions for use (IFU) to complete the HIVST and upload results. Trained healthcare workers (HCWs) observed and recorded any deviations from the IFU, and conducted a post-test survey to assess acceptability. Feasibility was evaluated by the number of participants who agreed to participate, completed the self-test, and uploaded all information onto the app correctly.

Results: Most participants $(98.7 \%)$ found the app easy to use. To reduce difficulties related to the IFU $(26 ; 8.7 \%)$, participants suggested multimedia supplements $(4 ; 1.3 \%)$, additional languages $(4 ; 1.3 \%)$ and simplified instructions $(5 ; 1.7 \%)$. All individuals approached, agreed to participate, $267(89.0 \%)$ correctly completed all steps and $210(78.7 \%)$ successfully captured all information on the app. Most errors $(26 ; 8.7 \%)$ were testing errors and $1(0.3 \%)$ was from the app sequence. Twelve (4.5\%) errors were with test strip imaging and 72 (27.0\%) discordances were with demographic information.

Conclusion: Despite some challenges with IFU interpretation and data capture via the app, this pilot showed that the Aspect ${ }^{\mathrm{TM}}$ HIVST app is an acceptable way to upload mobile HIVST results and demographic information to a central database.

Keywords: HIV self-test; digitisation; mobile app; monitoring and evaluation; digital health.

\section{Introduction}

In 2012, the OraQuick ADVANCE Rapid HIV-1/2 Antibody Test (OraSure Technologies Inc, Bethlehem, USA) was the first HIV self-test (HIVST) approved for sale in the United States as an over-the-counter HIVST rapid diagnostic test (RDT) for individuals with no prior HIV testing experience. ${ }^{1}$ Since then, over 2.5 million HIVST kits have been sold globally and more than 4 million have been distributed through donor funded programmes. ${ }^{2}$ The World Health Organization (WHO) strongly recommends that HIVST be utilised as a way to complement existing HIV services $^{3}$ as self-testing may reduce barriers associated with traditional facility-based testing, like travel, wait times and privacy concerns..$^{4,5}$

Based on this growing body of evidence, South Africa became one of over 40 countries to have incorporated HIV self-testing into their national HIV policies, ${ }^{6,7}$ with self-testing introduced as a way to help close the gap between the $84.9 \%$ of adults living with HIV who know their HIV status and the $90 \%$ target of the UNAIDS 90-90-90 initiative. ${ }^{5,8,9,10}$ The introduction of HIVST programmes will improve access to further HIV diagnostic services, prompting an increase in testing uptake and frequency, which could lead to earlier diagnosis. ${ }^{11}$

There are, however, several concerns related to HIVST, as there is no formal pipeline for users to self-report their results or be linked to care following the self-test. These HIVST kits are not diagnostic, but rather considered tests for triage, and all positive results should prompt the user 
to seek confirmatory testing by a trained healthcare professional. ${ }^{12}$ Furthermore, the independence of HIVST presents considerable challenges surrounding the monitoring and evaluation (M\&E) of HIVST programmes, which are required by public health stakeholders to understand the uptake and effectiveness. ${ }^{13}$

Strong mobile phone penetration in low- and middle-income countries (LMIC) $)^{14,15}$ has led to the development of a variety of mobile health (mHealth) interventions to complement HIVST. These include telephone hotlines, short message service interventions, internet-based platforms and mobile applications (apps). ${ }^{16,17,18,19,20}$ A Brazilian study conducted in 2019 showed that an internet-based intervention targeting men who have sex with men led to $21.4 \%$ of online participants self-reporting, whilst an interactive voice response telephone line in South Africa was found to link $9.8 \%$ of participants to care..$^{21}$ Whilst these platforms have shown varied success, the introduction of mHealth interventions for linkage to care and M\&E are in line with the South African National Department of Health mHealth Strategy (2015), and should be explored further. ${ }^{22}$

Despite data concerns in $\mathrm{LMICs}^{23}$ recent trends are towards the development of downloadable apps due to their agility and scalability. ${ }^{24}$ The app interface also provides developers with a malleable platform that can be tailored to individual users, allowing them to curate a collection of HIVST information, resources and guidance for testers, whilst also capturing the HIVST result data. ${ }^{19,20}$ Recently, HIVSmart ${ }^{\mathrm{TM}}$, a Canadian app, was developed to guide users through the testing process, link them to care, and store the HIVST result data. Preliminary evaluations in key Canadian populations, as well as healthcare workers in South Africa have shown the app to be feasible and acceptable; however, neither HIVSmart ${ }^{\mathrm{TM}}$, nor any other app, has been developed or tested for the general population in LMICs. ${ }^{9,20,25}$

South Africa has shown previous acceptance of HIV-related mHealth interventions with SmartLink, an app that improved linkage to care for clinic-based HIV testing in participants under 30 years of age. ${ }^{26}$ Another successful mHealth intervention, MomConnect, has been used by over 2 million pregnant South African women with information regarding their pregnancy, whilst also creating a national pregnancy registry. ${ }^{27,28}$

The Aspect ${ }^{\mathrm{TM}}$ HIVST app was developed to help strengthen and complement HIVST programmes by supporting selftesters through testing, facilitating linkage to care and digitising the reporting of HIVST results through an operational dashboard for M\&E. The specific objective of this pilot study was to evaluate the acceptability and feasibility of the Aspect ${ }^{\mathrm{TM}}$ HIVST app for individuals from the inner city of Johannesburg, in order to advise further scale-up. We present the findings from this pilot.

\section{Methods Study design}

This evaluation was a cross-sectional pilot study that ran for four weeks in July 2018. A convenience sample of 300 consenting adults was recruited from inner-city Johannesburg, South Africa. Recruitment was based around the Hillbrow Health Clinic by trained healthcare workers (HCW) who went into the surrounding communities and spoke to the public about the current study. Those interested were screened against inclusion/exclusion criteria, then brought to the Hillbrow Clinic to provide consent and complete the study. Participants were included if they owned a mobile phone (feature phones, or higher, for app compatibility) and could provide a valid mobile phone number, were 18 years or older, able to read English and able to provide written informed consent. Participants were excluded if they did not meet the inclusion criteria, were currently on a pre-exposure prophylaxis (PrEP) regime or any HIV treatment medication, could not provide valid identification or had any condition that may have interfered with the testing process (such as intoxication or poor vision).

\section{Development of the Aspect ${ }^{\mathrm{TM}}$ HIV-self-testing mobile app}

The Aspect ${ }^{\mathrm{TM}}$ HIVST app was designed for Android and deployed by SystemOne, LLC (Northampton, MA, USA), a diagnostic connectivity and disease intelligence company. The Aspect ${ }^{\mathrm{TM}}$ HIVST app was designed to be integrated with the existing Aspect ${ }^{\mathrm{TM}}$ software platform, a system designed to integrate directly with diagnostic instruments in order to collect digital results for real-time monitoring and reporting via an operational dashboard. The Aspect ${ }^{\mathrm{TM}}$ API can also communicate with RedCap, an existing South African healthcare database, and this application is already being used for reporting HIV viral load results and early infant HIV diagnosis (EID).

The Aspect ${ }^{\mathrm{TM}}$ HIVST app was developed using Dimagi Commcare (Washington, USA), a common data-gathering platform. The app was structured to allow the self-tester to collect their own demographic information, provide the tester with instructions on how to perform self-testing, input their interpretation of the test result, and capture a photo of the HIVST strip (Figure 1). Demographic data were collected with one question per page and included the self-tester's age,

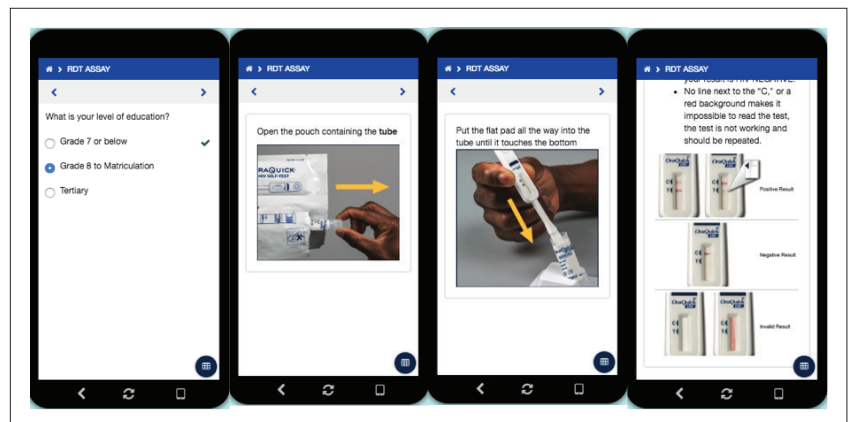

FIGURE 1: Screenshots of the Aspect ${ }^{\mathrm{TM}}$ HIV-self-testing mobile app. ${ }^{29}$ 
gender, mobile number, education level and whether they had self-tested before. The instructions, which were developed in English, provided the tester with step-by-step guidance, presented pictorially with simple wording taken directly from the HIVST kit manufacturer's instruction sheet, so that self-testing could be performed independently of a clinical setting.

All data gathered by the app was automatically uploaded via a secure server to the Aspect ${ }^{\mathrm{TM}}$ data management platform for viewing and review by the research team. Data collected in Aspect $^{\mathrm{TM}}$ was presented in aggregate form on a data dashboard that could be configured to display any relevant statistics for the research team. The app security was implemented with privacy by design methodology as per Protection of Personal Information (POPI) guidelines ${ }^{30}$ with patient data encrypted in transit and at rest, and also followed best practice guidelines in accordance with General Data Protection Regulation recommendations. ${ }^{31}$

\section{Data collection}

Trained HCWs obtained voluntary informed consent from the participant in a private room, then uploaded the participant's unique study identification number on the app. Once uploaded, the participant was handed a Samsung J5 smartphone, preloaded with the Aspect ${ }^{\mathrm{TM}}$ HIVST app, and an accompanying HIVST kit. The sealed test kit contained an English brochure with instructions for use (IFU) as part of the standard packaging; however, the participant was requested to perform the HIVST by following the IFU included in the HIVST kit and the digital version of the IFU provided on the app. Obtaining the sample takes 5-8 min when using the IFU (either paper or digital), followed by a 20 min incubation period. The OraQuick HIVST kit (Orasure Technologies Inc., Bethlehem, USA) was used for the study as it had already undergone full evaluation and was approved for use in South Africa. ${ }^{32}$ In a private room at a clinic, participants were asked to navigate the app and perform the HIVST with no assistance, whilst the HCW observed the process and recorded any deviations from the app instructions. Following the test, the HCW asked the participant a number of questions to obtain feedback on the app design and willingness to use an app for HIVST in future.

After the $28 \mathrm{~min}$ test was completed, the participant returned the phone to the HCW, who then uploaded their professional interpretation of the HIVST result on the app. Regardless of the HIVST result, the HCW performed confirmatory testing using a commercial HIV rapid test (Advanced Quality, InTec Products, Inc., Xiaman, China). If the participant's self-test and HCW confirmatory tests were discordant, a third test was performed (Abon 1/2/O Tri-line, Abon Biopharm Hangzhou Co., Hangshou China). The HCW uploaded all results, as applicable, on the app for reporting purposes. Participants with HIV-positive results (based on the confirmatory testing) were referred to a clinic as per standard of care. ${ }^{7}$

\section{Evaluation of HIV-self-testing and mobile app usage}

\section{Acceptability outcomes}

The evaluation of mobile apps may provide challenges to researchers due to the nature of their varied users, objectives, interfaces and mobility. ${ }^{33}$ In many cases, app developers and researchers develop data collection tools that are app-specific, in order to explore concepts exclusive to their app. ${ }^{34,35}$ For this pilot study, a survey was developed to advise on the preliminary scale-up of the app, which looked at general acceptability and asked a set of closed-ended (yes/no) and open-ended questions, similar to the methodologies found in other mHealth app evaluations..$^{21,36}$ The survey collected participant demographic information and included questions on whether the app was easy to use; which steps, if any, were difficult to understand; would they use the app again; would they be willing to download this app in the future and if they had any suggestions to improve the app. The demographic information collected by the survey and recorded by the $\mathrm{HCW}$ was also used to reference the accuracy of data capture on the app.

\section{Feasibility outcomes}

Similar to acceptability, there is no universal measure for determining the feasibility of an app; however, the generally accepted formula for feasibility includes three criteria: the participant's acceptance of using the app, the ability of the participant to complete tasks on the app and the ability of the app to perform the required tasks. ${ }^{37}$ These variables inevitably change based on the functionality of the app and its intended users, and for this pilot the feasibility criteria were as follows:

- User acceptance of the app: The number of participants who agreed to use the app.

- Successful test completion using the app: The number of participants who completed the testing through the app without error (i.e. experiencing difficulties or asking the HCW for assistance).

- Success of data capture through the app: The number of participants who captured their demographic information (when compared to the original records collected by the $\mathrm{HCW}$ ), uploaded their interpreted test result and captured their test-strip images correctly.

The final feasibility score is then presented as a percentage of the final criteria. ${ }^{37}$

\section{Data analysis}

All data extracted from the survey questionnaire (paper based) were entered into an access controlled Excel spreadsheet. The quantitative data captured on Aspect ${ }^{\mathrm{TM}}$ were extracted into a separate access controlled Excel spreadsheet. Quality control checks involved a $10 \%$ randomised check comparing paper-based tools against data on the spreadsheet. This was performed by the quality control officer on a daily basis. All data were coded and then exported to Stata version 15.1 (StataCorp, USA) for descriptive 
analysis. Data were grouped into categories to define demographic characteristics, then presented as frequency counts and percentages.

\section{Ethical consideration}

Ethics approval was obtained from the University of the Witwatersrand Human Research Ethics Committee (reference number 180504). All participants provided informed consent and were compensated ZAR150 for their time.

\section{Results \\ Demographics}

Of the 300 participants, over two-thirds $(211 ; 70.3 \%)$ were younger than 36 years old, there were 134 (44.7\%) female participants and $231(77.0 \%)$ participants who were educated up to at least high school level. Only $35(11.7 \%)$ participants indicated that they had previously self-tested. This information is presented in Table 1.

\section{HIV test outcomes}

Forty-two (14\%) participants interpreted their self-test result as HIV positive; however, there were $5(1.7 \%)$ discordant interpretations between participants and HCWs (Table 2). Three (1.0\%) results were interpreted as positive by the HCW but were interpreted as either invalid $(1 ; 0.3 \%)$ or negative $(2$; $0.7 \%$ ) by the participant, and $2(0.7 \%)$ results were interpreted as negative by the HCW but interpreted as either indeterminate $(1 ; 0.3 \%)$ or positive $(1 ; 0.3 \%)$ by the participant. Manual review of these discordant test result images, on the Aspect $^{\mathrm{TM}}$ dashboard by a senior researcher, confirmed the HCW interpretation in all discordances. The confirmatory testing of all participants conclusively diagnosed 43 (14.3\%) as HIV positive, all of whom were referred to care by the HCW.

\section{Acceptability}

Nearly all participants $(296 / 300 ; 98.7 \%)$ found the Aspect ${ }^{\mathrm{TM}}$ HIVST app easy to use, when surveyed; however, 26 (8.7\%) participants experienced some difficulty working through

TABLE 1: Demographic characteristics. Sample size $=300$.

\begin{tabular}{lcc}
\hline Demographic & Frequency & Percentage \\
\hline Age & 105 & \\
$18-25$ years old & 106 & 35.0 \\
$26-35$ years old & 89 & 35.3 \\
Over 35 years old & & 29.7 \\
Sex & 134 & \\
Female & 166 & 44.7 \\
Male & & 55.3 \\
Highest level of education & 18 & \\
Grade 7 or less & 213 & 6.0 \\
Grade 8 to matric & 69 & 71.0 \\
Tertiary school & & 23.0 \\
Ever self-tested before & 35 & \\
Yes & 265 & 11.7 \\
No & & 88.3 \\
\hline
\end{tabular}

the testing steps as outlined in the app (Table 3). Almost all of the difficulties were related to the self-testing procedures, as $18(6.0 \%)$ participants had difficulty sliding the tube into the stand, eight (2.7\%) had difficulties swabbing their gums and three $(1.0 \%)$ stated that the instructions were not clear. Another four (1.3\%) participants had difficulty taking and uploading the picture of the test to the app. When asked for suggestions to make the app easier to use, five $(1.7 \%)$ participants recommended that the instructions and steps be clarified, whilst four (1.3\%) participants specifically suggested adding a multimedia component to the instructions. Another four (1.3\%) participants suggested that the app be available in local languages and two $(0.7 \%)$ participants stated that the phone memory requirements should be decreased. All but one (299/300; 99.7\%) participants were willing to use the app again and only two $(0.7 \%)$ participants stated that they would not be willing to download the app in the future.

\section{Feasibility}

The final feasibility score was 70.0\%. All 300 individuals approached for this study agreed to participate in the evaluation of the Aspect ${ }^{\mathrm{TM}}$ HIVST app (Table 4). Of the 300 participants, 267 (89.0\%) successfully completed the HIVST by following all of the steps on the app without error. The majority of errors $(26 ; 8.7 \%)$ came from participants performing the testing procedures incorrectly, after reading the instructions on the app, which included sliding the tube into the stand $(18 ; 6.0 \%)$ and swabbing the gums $(8 ; 2.7 \%)$. Another four (1.3\%) participants had difficulties with the language of the instructions, whilst eight (2.7\%) participants made errors interpreting their HIVST results and one participant $(0.3 \%)$ could not properly navigate the pages of the app.

Of the 267 participants who completed the testing (Table 4), $210(78.7 \%)$ participants successfully captured all information on the app. The most erroneous variable was previous testing history, where 34 (12.7\%) participants submitted information that did not correlate with what they stated to the HCW

TABLE 2: Human immunodeficiency virus (HIV) testing outcomes. Sample size $=300$.

\begin{tabular}{lcc}
\hline HIV test results & Frequency & Percentage \\
\hline HIVST participant interpretation & 42 & \\
HIV positive & 253 & 14.0 \\
HIV negative & 5 & 84.3 \\
Invalid & & 1.7 \\
HIVST HCW interpretation & 43 & \\
HIV positive & 254 & 14.3 \\
HIV negative & 3 & 84.7 \\
Invalid & & 1.0 \\
Interpretation discordance & 295 & \\
Correctly interpreted & 5 & 98.3 \\
Interpretation error & & 1.7 \\
HIV confirmatory testing & 43 & \\
HIV positive & 257 & 14.3 \\
HIV negative & & 85.7 \\
\hline
\end{tabular}

HIVST, HIV-self-testing; HCW, healthcare workers. 
TABLE 3: Acceptability outcomes. Sample size $=300$.

\begin{tabular}{|c|c|c|}
\hline Question & Frequency & Percentage \\
\hline \multicolumn{3}{|l|}{ Did you find the mobile app easy to use? } \\
\hline Yes & 296 & 98.7 \\
\hline No & 4 & 1.3 \\
\hline \multicolumn{3}{|c|}{$\begin{array}{l}\text { What steps in the app did you find difficult to } \\
\text { understand or follow, if any? } \dagger\end{array}$} \\
\hline Sliding the tube into the stand & 18 & 6.0 \\
\hline Swabbing the gums & 8 & 2.7 \\
\hline Taking/saving the picture & 4 & 1.3 \\
\hline Instructions were not clear & 3 & 1.0 \\
\hline No difficulties & 274 & 91.3 \\
\hline \multicolumn{3}{|c|}{$\begin{array}{l}\text { If you choose to self-test again, would you be willing } \\
\text { to use the app again to help guide you? }\end{array}$} \\
\hline Yes & 299 & 99.7 \\
\hline No & 1 & 0.3 \\
\hline \multicolumn{3}{|c|}{$\begin{array}{l}\text { If you choose to self-test again, would you be willing } \\
\text { to download the app to your own mobile phone? }\end{array}$} \\
\hline Yes & 298 & 99.3 \\
\hline No & 2 & 0.7 \\
\hline \multicolumn{3}{|c|}{$\begin{array}{l}\text { Do you have suggestions on how to make this app } \\
\text { easier to use? } \dagger\end{array}$} \\
\hline Add voice/video notes & 4 & 1.3 \\
\hline Add local languages & 4 & 1.3 \\
\hline Clarification of instructions and steps & 5 & 1.7 \\
\hline Decrease phone memory requirements & 2 & 0.7 \\
\hline No suggestions & 285 & 95.0 \\
\hline
\end{tabular}

$\dagger$, Values may not add up to $100 \%$ as variables are not mutually exclusive.

TABLE 4: Feasibility outcomes.

\begin{tabular}{lccc}
\hline Feasibility criteria & Sample size & Frequency & Percentage \\
\hline Agreed to use the app & $\mathbf{3 0 0}$ & & \\
No & & 0 & 0.0 \\
Yes & 300 & 300 & 100.0 \\
Successfully completed the test using the & & & \\
app $\dagger$ & & 1 & 0.3 \\
App errors & & 26 & 8.7 \\
Testing errors & & 4 & 1.3 \\
Language errors & & 8 & 2.7 \\
HIVST interpretation errors & $\mathbf{2 6 7}$ & 267 & 89.0 \\
Successful completion & & & \\
Successfully captured all information on & & 12 & 4.5 \\
the app? $\dagger$ & & 2 & 0.7 \\
Age discordance & & 12 & 4.5 \\
Gender discordance & & 34 & 12.7 \\
Education discordance & & 12 & 4.5 \\
Previous test discordance & & 210 & 78.7 \\
Illegible image captured & & $\mathbf{2 1 0}$ & $\mathbf{7 0 . 0}$ \\
\hline Successful upload & $\mathbf{3 0 0}$ & & \\
Feasibility & & & \\
\hline
\end{tabular}

$\dagger$, Values may not add up to $100 \%$ as variables are not mutually exclusive.

during the survey. The variables of age and highest level of education each had $12(4.5 \%)$ participants who exhibited discordance and there were also two $(0.7 \%)$ discordances with gender compared with $\mathrm{HCW}$-recorded data. Twelve (4.5\%) participants also uploaded an illegible image of the HIVST strip to the app.

\section{Discussion}

This pilot study is the first investigation of an mHealth app to enhance monitoring and evaluation of HIVSTs for individuals from the inner city of Johannesburg, and the findings from this pilot have established that participants showed high acceptability of the intervention, whilst also identifying challenges that can be targeted for improvement as the platform scales up. The high acceptability was similar to that of the HIVSmart ${ }^{\mathrm{TM}}$ app and a Brazilian internet-based intervention; however, these studies only evaluated the feasibility of using the app to link patients to care or increase testing uptake, respectively.

The Aspect ${ }^{\mathrm{TM}}$ HIVST app, instead, aimed to guide participants through the testing process, then upload the results to a central server for $M \& E$, and this additional layer of complexity has introduced more opportunities for user error. The majority of errors, however, were not as a result of the app functionality, but rather test usability and the IFU that guided the self-testing process. Errors stemming from the IFUs have been well documented in a number of HIVST studies, including ones from South Africa. ${ }^{38,39,40}$ Suggestions like clarifying the instructions, incorporating video or voice notes, and offering additional languages should all be taken into consideration, especially as more HIVSTs, each with specific IFUs, become available to the market. Some of these suggestions have already been implemented by other platforms, as the HIVSmart ${ }^{\mathrm{TM}}$ app is already available in both of Canada's national languages, and provides supplemental video content. ${ }^{20}$

There were a number of discrepancies between HCWrecorded and app-captured data on participant demographic information. There were also some difficulties in the uploading of the test strip photo via the app. A simple summary page, similar to that seen on a banking app, before completing a transaction, could provide the user with an opportunity to review their information before submitting it through the app. This additional checkpoint should help prevent any data entry errors. One variable, however, previous HIV testing history, had 34 (12.7\%) discordant entries between what the HCW recorded and what the app captured; all 34 entries reported never having HIV tested to the HCW, but were captured in the app as having previously tested. It is possible that privacy of the app has revealed an interviewer bias, where some participants may not have felt comfortable sharing sensitive information with the HCW, but felt free to do so through the app. Previous mHealth studies have also found that self-administered tools may decrease interview bias; ${ }^{41}$ however, further evaluation of this app and its users would be required before stating that the app is responsible for removing or decreasing this interviewer bias.

Some participants also had difficulty understanding how to take a picture of the test strip. When test images were reviewed on the Aspect ${ }^{\mathrm{TM}}$ dashboard, the images were quite variable in terms of quality. The purpose of this functionality was to allow a third party to manually review test images and flag potential discordant results for follow-up. However, similarly to other studies, ${ }^{20,42}$ we had high concordance between participant and HCW interpretation of the self-test and, thus, this step may not even be necessary if lay persons 
are able to interpret results as accurately as trained HCWs. In low bandwidth environments, the requirement to upload images may also incur additional data charges and may not be cost effective.

With the number of countries adopting HIVST policies being on the rise, the $M \& E$ of these programmes poses a unique set of challenges ${ }^{12}$ and measurement of uptake and effectiveness becomes difficult. The Aspect ${ }^{\mathrm{TM}}$ HIVST app facilitated the capture of HIVST data directly to an operational dashboard, namely Aspect ${ }^{\mathrm{TM}}$. This dashboard was developed by SystemOne and is currently being used to report tuberculosis and HIV viral load results from over 3000 diagnostic instruments across 43 countries. $^{29}$ For this study, the dashboard displayed very basic summary HIV statistics, a list of individual test results and also supported the downloading of automated reports. This could allow a programme manager to remotely monitor indicators such as uptake, demographics of the testing population, HIV positivity rates, invalid rates and improve reporting against key performance indicators. The functionality of the dashboard also allows for the pushing of automated SMS notifications directly to the tester based on their HIV result, which could be used to promote confirmatory testing and help link them to care. ${ }^{43}$ This is especially important for HIVST, as one of the problems with home testing is that people receiving a positive diagnosis are suddenly faced with a serious diagnosis and no immediate access to information, counselling or treatment resources. ${ }^{11}$ The feasibility of these dashboard features should be considered for future research.

Data concerns are also an important issue in South Africa, with previous mHealth studies highlighting data costs and phone memory as a barrier to entry. ${ }^{26,44}$ Future app development should focus on keeping storage requirements minimal to ensure that the app is available for as many individuals as possible. Furthermore, the necessity to upload images may also incur additional data charges and may not be affordable for all users.

\section{Limitations}

The study had several limitations. Convenience sampling from one sub-district from inner-city Johannesburg was used to recruit participants limiting the generalisability of the findings, and the compensation of participants may have accounted for the very high participation rate. Furthermore, the majority of participants were under 35 years old, which may have made it easier for them to navigate a mobile app as they may be more tech-savvy than older age groups. The Aspect $^{\mathrm{TM}}$ HIVST app was only available in English. It was also only tested on a Samsung phone, and it may not reflect the usability of the app on other phones owned by the general population, especially across different operating systems and memory capacities. The discordance between HCW-recorded and app-captured demographics may reflect an interviewer bias, whilst the process of testing in front of a HCW may have increased the number of forced errors due to the pressures of being observed. Performing the HIVST with the app in a clinic, with a HCW present, may also present bias, as the app is intended to be used independently of a clinic setting. Another limitation of the pilot process was that the HCWs did not record the participants' interpretation on paper and, thus, results discordance could not be verified, as was done for the other variables.

Although recent studies have introduced validated data collection tools for mHealth usability, ${ }^{45}$ at the time of this study, there were also no validated data collection tools to measure the acceptability and feasibility of mHealth apps for HIVST, hence the study-specific questions may not be used to reproduce these results in similar settings. Similarly, the use of only one HIVST kit and its accompanying IFU means that these results cannot be generalised across all HIVSTs, especially since many of the errors were related to the interpretation of the IFU.

\section{Conclusions}

With millions of HIVST kits distributed worldwide without adequate tracking, the need for M\&E of these kits is ever increasing. On an individual level, this may lead to better linkage to care and follow-up with patients and, on a national level, tracking can identify areas of need to optimise kit distribution, marketing and supplementary information. Despite some challenges with IFU interpretation and data capture via the app, this pilot study has shown that the Aspect ${ }^{\mathrm{TM}}$ HIVST app is an acceptable way to upload mobile HIVST results and demographic information to a central database.

\section{Acknowledgements}

The investigators would like to thank the study implementation team and the study participants.

\section{Competing interests}

N.G. works for SystemOne and was involved in the design of the Aspect HIVST app.

\section{Authors' contributions}

N.G., N.R., M.P. and M.M. designed the study. N.G., N.R. and M.P. collected data, N.G., N.R., M.P. and A.E.F. were involved in the data cleaning and analysis; A.E.F., S.T.L.-E. and N.G. wrote the initial draft of the manuscript. All authors critically reviewed and approved the final draft.

\section{Funding information}

Funding to carry out the research project has been obtained through a Gates Grand Challenges Award to SystemOne (grant number: OPP1182240) and Grand to Wits RHI received from the Bill and Melinda Gates Foundation (grant number OPP1132929).

\section{Data availability statement}

Data are available upon reasonable request. 


\section{Disclaimer}

The views and opinions expressed in this article are those of the authors and do not necessarily reflect the official policy or position of any affiliated agency of the authors.

\section{References}

1. Richter M, Venter WD, Gray A. Enabling HIV self-testing in South Africa. S Afr J Med. 2016;13(4):186-187. https://doi.org/10.7196/sajhivmed.858

2. Unitaid, World Health Organization. Market and technology landscape: HIV rapid diagnostic tests for self-testing. 4th ed. Geneva: Unitaid; 2018.

3. Wong $V$, Jenkins $E$, Ford $N$, Ingold $H$. To thine own test be true: HIV self-testing and the global reach for the undiagnosed. J Int AIDS Soc. 2019;22(S1):e25256. https:// doi.org/10.1002/jia2.25256

4. Figueroa C, Johnson C, Verster A, Baggaley R. Attitudes and acceptability on HIV self-testing among key populations: A literature review. AIDS Behav. 2015;19(11):1949. https://doi.org/10.1007/s10461-015-1097-8

5. Makusha T, Knight L, Taegtmeyer M, et al. HIV self-testing could 'revolutionize testing in South Africa, but it has got to be done properly': Perceptions of key stakeholders. PLoS One. 2015;10(3):e0122783. https://doi.org/10.1371/journal. pone. 0122783

6. World Health Organization. Number of countries adopting HIV self-testing policies rises sharply [homepage on the Internet]. 2020 [updated 2017 Jul 25; cited 2020 Jan 15]. Available from: https://www.who.int/hiv/mediacentre/news/hiv-selftesting-increases/en/

7. National Department of Health South Africa. National HIV self screening guidelines 2018. Pretoria: Department of Health Republic of South Africa; 2018.

8. AIDS. Global AIDS update. UNAIDS [homepage on the Internet]. 2020 [cited 2020 Feb 1]. Available from: http://www.unaids.org/en/resources/documents/2017/\%20 2017_data_book

9. Pai NP, Behlim T, Abrahams L, et al. Will an unsupervised self-testing strategy for HIV work in health care workers of South Africa? A cross sectional pilot feasibility study. PLoS One. 2013;8(11):e79772. https://doi.org/10.1371/journal.pone.0079772

10. Human Sciences Research Council. The Fifth South African National HIV prevalence, incidence, behaviour and communication survey, 2017: HIV impact assessment summary report. Pretoria: Human Sciences Research Council: 2018.

11. Ritchwood D, Selin A, Pettifor A, et al. HIV self-testing: South African young adults' recommendations for ease of use, test kit contents, accessibility, and supportive resources. BMC Public Health. 2019;19(1):123. https://doi.org/10.1186/s12889019-6402-4

12. Venter F, Majam M, Jankelowitz L, et al. South African HIV self-testing policy and guidance considerations. Southern Afr J HIV Med. 2017;18(1):775. https://doi. org/10.4102/sajhivmed.v18i1.775

13. Fionet: Mobile diagnostics integrated with cloud information services. USAID mHealth compendium, Volume 2; pp. 62-63 [homepage on the Internet]. 2017 [cited 2019 Nov 13]. Available from: http://www.africanstrategies4health.org/ uploads/1/3/5/3/13538666/fionet_-_mobile_diagnostics.pdf

14. GSMA. The mobile economy Sub-Saharan Africa. London: GMSA; 2017.

15. Wedderburn CJ, Murtagh M, Toskin I, Peeling RW. Using electronic readers to monitor progress toward elimination of mother-to-child transmission of HIV and monitor progress toward elimination of mother-to-child transmission of HIV and syphilis: An opinion piece. Int J Gyn
doi.org/10.1016/j.ijgo.2015.04.006

16. Mokgatle MM, Madiba S. High acceptability of HIV self-testing among technical vocational education and training college students in Gauteng and North West Province: What are the implications for the scale up in South Africa? PLoS One. 2017;12(1):e0169765. https://doi.org/10.1371/journal.pone.0169765

17. Majam M, Quaife $M$, Phatsoane $M$, et al. High self-reporting of HIV self-test results through an interactive voice response telephone line in inner city Johannesburg. Poster session presented at: IAS Conference on HIV Science, Mexico; 2019 Jul 21-24.

18. De Boni RB, Lentini N, Santelli AC, et al. Self-testing, communication and information technology to promote HIV diagnosis among young gay and other men who have sex with men (MSM) in Brazil. J Int AIDS Soc. 2018;21(5):e25116. https://doi.org/10.1002/jia2.25116

19. Janssen R, Engel N, Esmail A, et al. Alone but supported: A qualitative study of an HIV self-testing app in an observational cohort study in South Africa. AIDS Behav. 2020;24(2):467-474. https://doi.org/10.1007/s10461-019-02516-6

20. Pai PN, Smallwood M, Desjardins L, et al. An unsupervised smart app-optimized HIV self-testing program in Montreal, Canada: Cross-sectional study. J Med Internet Res. 2018;20(11):e10258. https://doi.org/10.2196/10258

21. De Boni RB, Veloso VG, Fernandes NM, et al. An internet-based HIV self-testing program to increase HIV testing uptake among men who have sex with men in Brazil: Descriptive cross-sectional analysis. J Med Internet Res. 2019;21(8):e14145. https://doi.org/10.2196/14145
22. National Department of Health, South Africa. mHealth Strategy 2015-2019. Pretoria: National Department of Health; 2015.

23. ResearchICTafrica.net. Cape Town: Research ICT Africa; 2017. State of prepaid market in South Africa: Submission to parliament of South Africa on the cost to communicate in South Africa [homepage on the Internet]. 2016 [cited 2019 Dec 8]. Available from: https://www.ellipsis.co.za/wpcontent/uploads/2016/09/cost to communicate research ict africa.pdf

24. Gough A, Hunter RF, Ajao O, et al. Tweet for behavior change: Using social media for the dissemination of public health messages. JMIR Public Health Surveill. 2017;3(1):e14. https://doi.org/10.2196/publichealth.6313

25. McGill University Health Centre Foundation. HIV Smart App [homepage on the Internet]. 2016 [cited 2020 Jan 12]. Available from: https://www.muhcfoundation. com/current-projects/hiv-smart-app/

26. Venter WDF, Fischer A, Lalla-Edward ST, et al. Improving linkage to and retention in care in newly diagnosed HIV-positive patients using smartphones in South Africa: Randomized controlled trial. JMIR Mhealth Uhealth. 2019;7(4):e12652. https://doi.org/10.2196/12652

27. Seebregts C, Dane P, Parsons AN, et al. Designing for scale: Optimising the health information system architecture for mobile maternal health messaging in South Africa (MomConnect). BMJ Glob Health. 2018;3(S2):e000563. https://doi. org/10.1136/bmjgh-2017-000563

28. Praekelt. MomConnect for nurses and midwives programme status report (Quarter 2) 1 April 2017-30 June. Johannesburg: Praekelt; 2017.

29. SystemOne. Aspect, see what matters [homepage on the Internet]. 2020 [cited 2020 Mar 24]. Available from: https://systemone.id/aspect

30. Republic of South Africa. Government Gazette, Act No 4 of 2013: Protection of Personal Information Act, 2013. Cape Town: The South African Government; 2013.

31. The EU General Data Protection Regulation (GDPR) (Regulation (EU) 2016/679). European Parliament and Council [homepage on the Internet]. 2016 [cited 2019 Nov 3]. Available from: http://www.eugdpr.org/

32. Ingold $\mathrm{H}$, Mwerinde $\mathrm{O}$, Ross AL, et al. The Self-Testing AfRica (STAR) Initiative: Accelerating global access and scale-up of HIV self-testing. J Int AIDS Soc. 2019; 22(S1):e25249. https://doi.org/10.1002/jia2.25249

33. Baharuddin R, Singh D, Razali R. Usability dimensions for mobile applications A review res. J. Appl Sci Eng Technol. 2013;5(6):2225-2231. https://doi. org/10.19026/rjaset.5.4776

34. Reynoldson C, Stones C, Allsop M, et al. Assessing the quality and usability of smartphone apps for pain self-management. Pain Med. 2004;15(6):898-909. https://doi.org/10.1111/pme.12327

35. Jake-Schoffman DE, Silfee VJ, Waring ME, et al. Methods for evaluating the content, usability, and efficacy of commercial mobile health apps. JMIR Mhealth Uhealth. 2017;5(12):e190. https://doi.org/10.2196/mhealth.8758

36. Birkhoff S, Cantrell MA, Moriarty H, Lustig R. The usability and acceptability of a patient-centered mobile health tracking app among a sample of adult radiation oncology patients. ANS Adv Nurs Sci. 2018:(41)3:243-259. https://doi. org/10.1097/ANS.0000000000000202

37. Pai NP, Bhrgava $M$, Joseph $L$, et al. Will an unsupervised self-testing strategy be feasible to operationalize in Canada? Results from a pilot study in students of a large Canadian University. AIDS Res Treat. 2014;2014(747619):1-8. https://doi. org/10.1155/2014/747619

38. Simwinga M, Kumwenda MKRD, Kayira L, et al. Ability to understand and correctly follow HIV self-test kit instructions for use: Applying the cognitive interview technique in Malawi and Zambia. J Int AIDS Soc. 2019;22(S1):e25253. https://doi. org/10.1002/jia2.25253

39. Smith P, Wallace M, Bekker LG. Adolescents' experience of a rapid HIV self-testing device in youth-friendly clinic settings in Cape Town South Africa: A cross-sectional community based usability study. JIAS. 2016;19(1):28406597. https://doi. org/10.7448/IAS.19.1.21111

40. Majam M, Mazzola L, Rhagnath N, et al. Usability assessment of seven HIV selftest devices conducted with lay-users in Johannesburg, South Africa. PLoS One. 2020;15(1):e0227198. https://doi.org/10.1371/journal.pone.0227198

41. $\operatorname{Kim~} \mathrm{H}$, Xie B. Health literacy and internet- and mobile app-based health services: A systematic review of the literature. Proc Assoc Info Sci Tech. 2015;52(1):1-4. https://doi.org/10.1002/pra2.2015.145052010075

42. Bwana P, Ochieng L, Mwau M. Performance and usability evaluation of the INST HIV self-test in Kenya for qualitative detection of antibodies to HIV. PLoS One. 2018;13(9):e0202491. https://doi.org/10.1371/journal.pone.0202491

43. Siedner M, Santorino D, Lankowski A, et al. An SMS intervention to improve HIV linkage to care: A randomized, comparative effectiveness trial. 2014 Presented at: 21st Conference on Retroviruses and Opportunistic Infections; 2014 March Boston, MA.

44. Fischer AE, Sebidi J, Barron P, Lalla-Edward ST. The MomConnect nurses and midwives support platform (NurseConnect): A qualitative process evaluation. JMIR Mhealth Uhealth. 2019;7(2):e11644. https://doi.org/10.2196/11644

45. Zhoe L, Bao J, Setiawan MA, Saptono A, Parmanto B. The mHealth app usability questionnaire (MAUQ): Development and validation study. JMIR Mhealth Uhealth. 2019;7(4):e11500. https://doi.org/10.2196/11500 